\title{
Agôn
}

Revue des arts de la scène

\section{2| 2009}

L'accident

\section{Présentation de l'Emupo : de l'accidentel au chaotique}

\section{Les Emeudroïdes}

\section{(2) OpenEdition \\ Journals}

Édition électronique

URL : http://journals.openedition.org/agon/1044

DOI : 10.4000/agon. 1044

ISSN : 1961-8581

Éditeur

Association Agôn

Référence électronique

Les Emeudroïdes, «Présentation de l'Emupo : de l'accidentel au chaotique », Agôn [En ligne], 2 | 2009, mis en ligne le 15 décembre 2009, consulté le 02 juin 2020. URL : http://journals.openedition.org/ agon/1044; DOI : https://doi.org/10.4000/agon.1044 


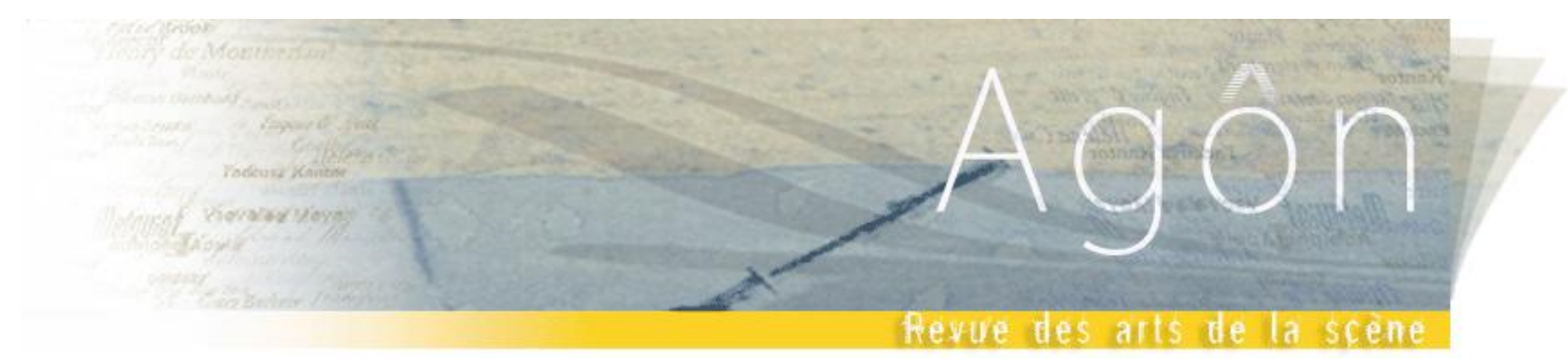

Présentation de l'Emupo : de l'accidentel au chaotique

La création de l'Emupo mobilise notre ensemble d'improvisation «Les Emeudrö̈des » (Clément Canonne, Roméo Monteiro, Nicolas Nageotte et Joris Rühl) depuis maintenant plus de deux ans. Nous présentons rapidement l'Emupo avant d'en revenir au lien fort qu'il entretient avec la notion d'accident.

L'Emupo est une interface logicielle destinée à l'improvisation, dont l'idée a germé lors de notre formation au département de composition du Conservatoire National Supérieur de Musique et de Danse de Lyon. Il s'agissait par là de renouveler notre jeu improvisé, notamment :

- En introduisant un nouveau partenaire dans nos interactions, l'Emupo, dont on verra par la suite de quelles manières il peut stimuler notre jeu instrumental par ses «propositions ».

- En utilisant les possibilités intrinsèques de l'Emupo pour nous aider à penser autrement l'articulation collective de la forme lors de nos séances d'improvisation collective.

D'autre part, nous souhaitions pouvoir utiliser l'Emupo tant de manière autonome qu'en relation avec notre jeu instrumental, voire en relation avec le jeu d'autres improvisateurs.

Il fallait pour cela éviter que cette aventure ne prenne la forme, presque paradigmatique aujourd'hui dans le domaine des musiques mixtes (mêlant instruments acoustiques et informatiques), du traitement en temps réel ${ }^{1}$. Il n'y a donc aucune relation (et notamment aucune relation algorythmique) entre le jeu de l'Emupo et le jeu des instrumentistes s'exprimant éventuellement en sa compagnie. Pour le dire simplement, l'Emupo est comme tous les instruments : s'il n'a pas besoin d'informations sonores entrantes pour produire du son, il doit en revanche être contrôlé par un musicien, seul à même de garantir la pertinence de l'interaction entre sa production sonore et celle des autres improvisateurs.

Néanmoins, une spécificité de taille distingue l'Emupo des autres instruments : cet instrument produit en effet un unique discours musical, capable d'autonomie, mais contrôlé simultanément par plusieurs musiciens (souvent les quatre membres des Emeudroides). La nature des interactions musicales en est ainsi profondément renouvelée, en ajoutant au niveau d'interaction inter-instrumental (instruments/instruments mais aussi instruments/Emupo, en tant qu'il est également un instrument) ${ }^{2}$ un niveau intra-instrumental (le pilotage collectif de l'Emupo proprement dit).

Mais à vrai dire, c'est davantage un méta-contrôle que nous opérons sur l'Emupo. En effet, l'Emupo produit continuellement de nouveaux événements sonores (cela ne veut pas dire qu'il y a tout le temps du son, puisque le silence est également un événement sonore) : ce flux d'évènements généré par l'ordinateur place l'utilisateur dans une attitude musicale

\footnotetext{
1 Traitement du signal instrumental par le programme informatique, qui récupère ce signal pour ensuite diffuser un ensemble d'événements sonores basés, d'une manière ou d'une autre, sur ce signal entrant.

${ }^{2}$ En supposant bien sûr qu'une improvisation instrumentale se superpose à l'improvisation de l'Emupo.
} 
foncièrement différente de celle de l'instrumentiste : dans l'impossibilité de déclencher un son, celui-ci doit plutôt penser son action en termes de modelage du matériau musical. L'utilisateur peut ainsi réaliser ce modelage du flux continu en manipulant une série de paramètres musicaux (hauteurs/ intensités/ durées/ rythmicité/ spatialisation/ timbre/ effets...), qui sont gérés par diverses courbes de distribution de probabilités, laissant une place plus ou moins grande à l'accident.

Le pilotage de l'Emupo se faisant, dans sa version première, sans quitter nos instruments habituels (clarinettes, percussions, piano), il a fallu, pour d'évidentes raisons d'ergonomie, personnaliser les interfaces de contrôle (joysticks, rotatifs, capteurs...) pour permettre à chacun d'accéder aux multiples fenêtres (d'édition, de sélection, d'interpolation...) que comporte le programme.

Le matériau sonore que travaille l'Emupo est issu d'enregistrements de nos propres improvisations instrumentales, classées selon divers critères acoustiques, organologiques ou musicaux (lisse/granulaire, brillance, modes de jeu, linéarité, variabilité...). Ceci nous permet de pallier dans une certaine mesure l'absence d'interaction « temps-réel » entre l'Emupo et notre jeu instrumental, en créant des effets de trompe l'œil, d'écho, d'imitation, relativement efficaces, entre les sons d'origine acoustique et les sons électroniques. Cela contribue également à homogénéiser les différents niveaux de discours qui interviennent lors d'une production avec l'Emupo.

On entend dans l'extrait présenté ici (qui est de l'Emupo solo : c'est-à-dire que nous nous contentons de piloter celui-ci et que nous ne jouons pas de nos instruments acoustiques en plus) comment les nombreux accidents qui émaillent le discours nous permettent de construire une improvisation relativement structurée, aux sections et transitions assez clairement articulées (cf. l'article, dans ce même numéro, "Quelques réflexions sur Improvisation et Accident »). La programmation de l'Emupo rend particulièrement propice cette émergence d'accidents pour deux raisons distinctes : 1'Emupo est un instrument utilisant des processus aléatoires contrôlés ; et l'Emupo est un instrument chaotique.

Nous pouvons exercer un contrôle plus ou moins déterministe sur l'Emupo : en effet, plusieurs paramètres (hauteurs, intensités, durées) sont régis par des courbes de distribution de probabilité. Par exemple, nous pouvons décider de travailler dans une zone de hauteurs medium : nous serons alors certains que tous les sons produits par l'Emupo seront restreints à un certain ambitus, déterminé par nous ; et les sons seront donc relativement peu transposés, proches de leurs timbres et durées originales (c'est-à-dire tels que nous les avons enregistrés en studio). Mais le joueur qui est en charge du contrôle des hauteurs peut décider d'introduire une possibilité d'accident : par exemple un son très aigu au milieu de tous ces sons médium. Il peut alors éditer d'un simple geste la courbe de distribution des probabilités concernant la hauteur de telle façon qu'il y ait 5\% de chance pour qu'un son très aigu soit produit. Cela signifie qu'à chaque fois que la machine tire un nouvel événement sonore, celui-ci sera dans un registre médium dans $95 \%$ des cas, mais qu'il y aura toujours $5 \%$ de chance pour que le son tiré soit une transposition très aigue du son d'origine. Il y a donc un contrôle de l'accident, c'est-à-dire qu'un joueur peut décider d'introduire la probabilité d'un accident ; en même temps, dans la plupart des cas (c'est-à-dire sauf si le joueur décide d'attribuer $100 \%$ de probabilité au nouvel événement), cela reste un accident, c'est-à-dire un événement imprévisible (ce qui est le propre d'un événement dont la probabilité d'advenir est fort basse). 


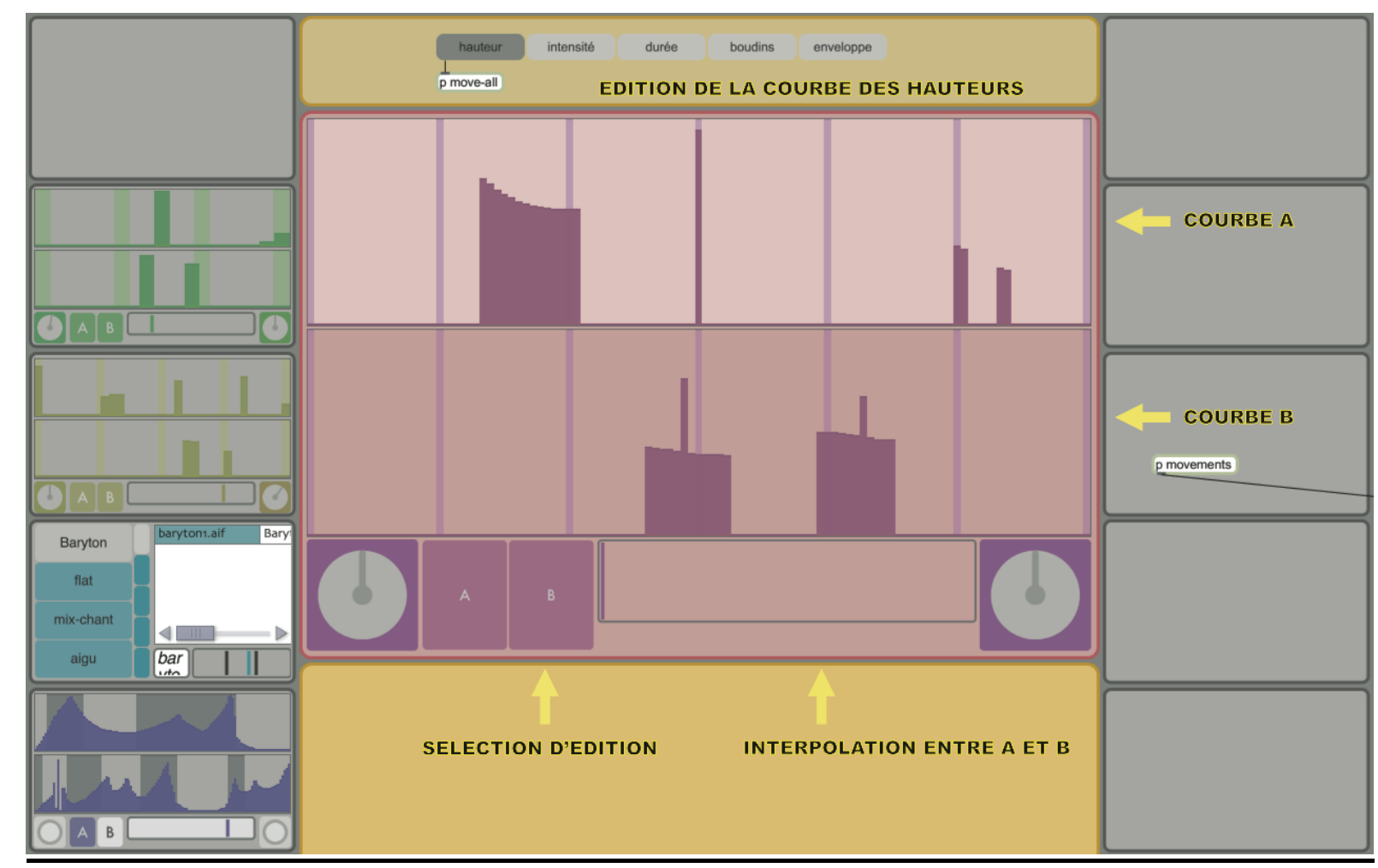

Exemple de la fenêtre d'édition de la courbe des hauteurs. Le joueur peut éditer deux courbes : il s'agit de courbes discrètes de distribution de probabilités. L'axe des abscisses indique la valeur du paramètre en question (du grave à gauche vers l'aigu à droite); l'axe des ordonnées indique la valeur relative de la probabilité d'occurrence de la valeur du paramètre. Le joueur peut également interpoler entre ces deux courbes. Il peut enfin opérer une translation d'axe $x$ sur chacune de ces courbes (cela revient à effectuer une transposition dans le cas de la courbe des hauteurs).

Ce qui apparaît dans les fenêtres en format réduit sur les côtés de l'écran, c'est l'état actuel de tous les paramètres que ne contrôle pas le joueur considéré. S'il désire changer de rôle (c'est-à-dire travailler sur d'autres paramètres), il peut simplement sélectionner une de ces fenêtres pour « prendre la main » sur ce paramètre (à condition que ce dernier ne soit pas déjà contrôlé par un autre joueur). Le paramètre sélectionné apparaît alors dans la fenêtre centrale

Néanmoins, les choses sont en réalité beaucoup plus compliquées que cela. En effet, tous ces paramètres, modifiés en temps réels par les différents joueurs contrôlant l'Emupo, sont étroitement interdépendants : il y a notamment des effets de contraintes rétroactives entre le paramètre de la rythmicité (l'organisation des événements sonores dans le temps : il s'agit, pour faire simple, des idées de mesure ou de carrure) et les autres paramètres pouvant aboutir à court-circuiter ce contrôle précis des probabilités d'accident effectués par certains joueurs. D'une manière générale, l'interdépendance des paramètres est telle que le comportement de l'Emupo est assez largement chaotique (ce qui est très différent d'un comportement aléatoire) : ainsi, non seulement un joueur ne peut jamais être absolument certain de l'impact musical qu'aura son geste (il s'agit donc d'un geste éminemment anti-instrumental : il n'y a pas de traduction entre gestes et sons, ce qu'impliquent la conception en termes de métacontrôle et l'existence de contraintes rétroactives), mais encore une très légère variation de l'état de l'Emupo peut avoir des conséquences musicales extrêmement importantes. Ainsi en 
termes de timbre : bien qu'une personne puisse contrôler spécifiquement le choix du timbre, les différents mixages de timbre (il associe en fait trois échantillons à la suite qui constituent, ensemble, le matériau travaillé par l'Emupo) et la position à partir de laquelle les échantillons seront lus, comme presque tous les paramètres contrôlés ont une influence sur la nature du timbre effectivement produit, une légère transposition vers l'aigu par la personne s'occupant du choix des hauteurs peut par exemple suffire à modifier fortement le timbre final produit par l'Emupo ; pour le dire autrement, l'Emupo est très sensible aux conditions initiales de détermination du son : une légère modification dans ces conditions entraîne des conséquences difficilement anticipables.

L'improvisation avec l'Emupo prend tous son sens quand ce dernier est confronté avec un jeu instrumental ; l'Emupo oblige en effet les improvisateurs à éviter toute solution « de confort », en rendant son sens étymologique à l'improvisation : quelque chose d'imprévu. L'Emupo nous suggère de l'inhabituel, bien sûr, tant sa "pâte sonore » nous invite à de nouvelles explorations instrumentales, inédites pour nous. Mais l'Emupo nous propose aussi de l'imprévu, de l'accident, dont nous pouvons nous saisir pour renouveler telle ou telle proposition instrumentale, opérer un brusque changement d'orchestration, amener une transition vers une nouvelle séquence... En même temps, nous sommes très loin de la musique aléatoire, ou de la dépendance servile à la machine, puisque ce sont nous-mêmes qui introduisons diverses possibilités d'accidents, qui rendons la machine plus ou moins instable. L'Emupo reste un instrument, même s'il a été spécifiquement conçu comme un outil d'improvisation collective : et comme tous les instruments, il nécessite une longue pratique, et beaucoup de travail pour que le musicien puisse obtenir un résultat digne d'intérêt en improvisant.

\section{Pour citer ce document}

«Présentation de l'Emupo : de l'accidentel au chaotique», Agôn [En ligne], N² : L'accident, Dossiers, mis à jour le : 23/11/2009, URL : http://w7.ens-lsh.fr/agon/index.php?id=1044. 